\title{
The Electrical Characteristics Investigation of the Module-Level Miniaturization with Embedded Device Technology
}

\author{
Shuji Sagara and Masaya Tanaka \\ Dai Nippon Printing Co., Ltd. 2-2-1 Fukuoka, Fujimino-shi, Saitama, 356-8507, Japan
}

(Received August 19, 2009; accepted November 9, 2009)

\begin{abstract}
Previously, we developed a high-density assembly technology to miniaturize the motion control CPU SiP for humanoid robots. It is necessary to mount a number of passive components on an expensive buildup PWB to accomplish this CPU $\mathrm{SiP}$ assembly and satisfy electrical performance requirements. As a result, we developed an embedded device technology to realize further miniaturization and achieve excellent electrical performance with the CPU SiP. This paper is intended as an investigation of the impacts of module-level miniaturization with embedded active device technology on the electrical performance. From our investigation, it is concluded that the CPU-embedded SiP has excellent electrical performance, such as signal reflection, cross-talk noise and simultaneous switching output noise. In addition, the CPUembedded SiP has sufficient signal transmission properties for a data rate of $4 \mathrm{Gbps}$. The results of this investigation will change the future technology position of module-level miniaturization with embedded device technology.
\end{abstract}

Keywords: Motion Control CPU Module, CPU SiP, Embedded Device Technology, High Speed Signal Transmission

\section{Introduction}

The authors developed a high-density assembly technology to miniaturize the motion control CPU module for humanoid robots, which supports hardware-level real-time processing functions and real-time communication. In this CPU module, a $10 \times 10 \mathrm{~mm}$ CPU die with 1,000 I/O terminals and four DDR packaged devices are mounted on a ten-layered $33 \times 33 \mathrm{~mm}$ buildup substrate. From this development, we checked that further miniaturization is impossible if only conventional surface mount technology is applied to this CPU module assembly, because the contact area of all the surface mount components and the surface area of the buildup substrate are almost the same. Therefore, the evolution of CPU modules for humanoid robots cannot be accomplished without innovations in novel three-dimensional assembly technologies. In other words, we demonstrated the result of an ultimate surface mount technology through this development of a CPU module for humanoid robots.[1]

Figure 1 shows a motion-control CPU module for humanoid robots, consisting of a M-RMT (Motion controlResponsive Multi-threaded) processor CPU, $256 \mathrm{MB}$ main memories, system boot ROM with chip-stacking technology, and peripheral packaged devices with surface mount

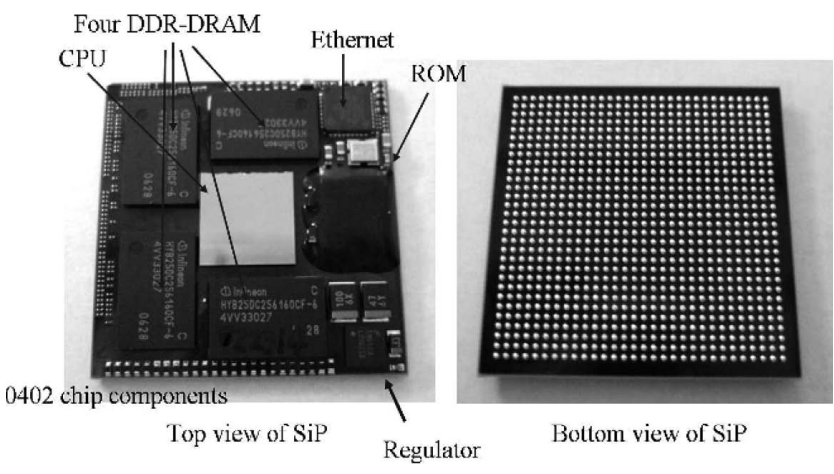

Fig. 1 Motion control CPU module for humanoid robots.

technologies. This motion control CPU module for humanoid robots was developed as the part of the NEDO-project. In this module, more than one hundred individual passive components are mounted on the $33 \times 33 \mathrm{~mm}$ substrate to keep the signal transmission qualities of the DDR in access mode and to stabilize the multiple power supply systems. It is very important that the cubic volume of the CPU module can be reduced without impairing electrical functions.

First level and second level three-dimensional [3D] integration technologies, such as TSV (Through Silicon Via), SOS (Silicon on Silicon) and POP (Package on Package) 
have many technical advantages in miniaturization. On the other hand, there are substantive problems in applying these technologies to mass production. On SOS and TSV technologies, signal qualities are not affected by the parasitic impedance of wiring-pattern, because the wiring-pattern lengths between LSIs are extremely short on these structures. On the other hand, both $3 \mathrm{D}$ integration technologies require an advanced thin-film fabrication technology and a super-connect technology to achieve a high-performance integrated system; additionally, their production costs depend greatly on the manufacturing yield of LSI fabrication. With POP technology, it is possible to ensure high manufacturing yields, because each package can be applied after the electrical inspection. However, the number of interconnections between LSIs will be strongly restricted, because the wiring-patterns and external pads can be arranged only in the peripheral region of the package plane surface. Thus, these 3D integration technologies have both merits and demerits. Therefore, we have developed a novel module-level 3D integration technology to overcome the weaknesses of these first and second level 3D integration technologies.

We first announced our findings on embedded passive and active device technologies using the $\mathrm{B}^{2}$ it PWB fabrication method at the International Symposium on Microelectronics in 2007.[2] In this symposium we showed only that the mechanical reliability of the passive and active device embedded PCB substrate was enough. In the present paper, we clarify and report on the electrical capabilities of embedded active device substrates through some feasibility research on the electrical performance with CPU and memory module configurations.

There are three main targets in this investigation. The first is to find how to embed the electrically-tested CPU into the PWB substrate. For high-performance devices with large numbers of pins, it is very difficult to execute electrical testing after completing the device-embedding process. It is necessary to draw out all terminals for the CPU function test on the top surface of the PWB substrate. Therefore, a complex wiring-pattern design is required to embed a CPU in a PWB substrate. We developed a thin testable package to resolve this serious issue, which can easily screen the electrical function before the $\mathrm{CPU}$ embedding process.

The second target is to find how to enhance the capabilities of electrical performance in a $3 \mathrm{D}$ space including the internal space of the PWB substrate. Embedded device technology can reduce the loading impedance of signal wiring pattern connecting the CPU to the memories. We verify the effect of this reduction in loading impedance on signal quality. Specifically, we verify memory access performance by means of a SPICE simulation method with a modeled circuit of accurate electrical parameters.

The third target is to find how to adequately improve the signal qualities of high-speed signals from the embedded $\mathrm{CPU}$, after passing through the wiring patterns of internal layers and vias. We evaluate the communication performance of the embedded active device substrate with the structure of a hybrid buildup substrate (i.e., build-up on PTH core) and core-less buildup substrate (i.e., all- $\mathrm{B}^{2}$ it substrate).

\section{CPU embed procedure with $B^{2}$ it fabrication}

In this chapter, we introduce the outlines of the CPU embed procedure with $\mathrm{B}^{2}$ it fabrication technology. [3] The $\mathrm{CPU}$ embed procedure consists of a packaging process for the bare $\mathrm{CPU}$ die, a soldering process to connect the CPU package with the base PWB substrate, and a laminate process to embed the $\mathrm{CPU}$ package using $\mathrm{B}^{2}$ it fabrication technology.

\subsection{Thin CPU packaging process}

We have developed a new testable thin CPU package to embed a high pin-count CPU die into the PWB substrate. This thin CPU package is composed of a $0.1 \mathrm{~mm}$ thick CPU die, a double-sided substrate with $0.04 \mathrm{~mm}$ thick dielectric, and encapsulation resin to cover the whole area of the CPU die. The total thickness of this finished thin CPU package without lead-free solder bumps is $0.23 \mathrm{~mm}$.

Figure 2 shows the completed thin CPU package with solder bumps, connected to the base PWB substrate. These lead-free solder bumps are directly attached to $\mathrm{Cu}$ raw external lands on the package back side, in order to prevent the formation of inter-metallic compounds by the diffusion of tin. On the other hand, Au stud bumps formed on $\mathrm{Al}$ pads of the CPU die are attached to the Cu-raw pads on the package substrate using a pressure welding method. The top of the substrate with the attached CPU is encapsulated in epoxy-resin-based composites with silica filler in order to reduce warpage during the IR reflow pro-
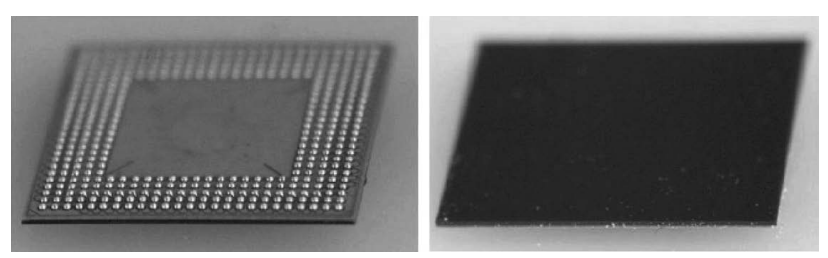

Fig. 2 Photograph of completed thin CPU package. 
cess. All the external pads of this thin CPU package are drawn on dielectric using the fan-in and fan-out shapes, and these are arranged in the pitch of the JEDEC standard formats. Therefore, it is possible to do a CPU electrical test with a commercial test socket developed for conventional CSP packages. From the development of this special thin CPU package, we have an easy technique to embed a CPU with 400 pins into a PWB substrate.

\subsection{Thin CPU package embed procedure with $\mathrm{B}^{2}$ it fabrication process}

Figure 3 shows the thin CPU package embed procedure using $\mathrm{B}^{2}$ it fabrication technology. Initially, a two- or fourlayered metal circuit PWB is prepared to mount the thin CPU package with lead-free solder bumps. The opposite face of this four layered metal circuit PWB (i.e., the back side of the CPU mounted surface) is used as the surfacemount area for the DDR memories.

Secondly, the electrically inspected thin CPU package is

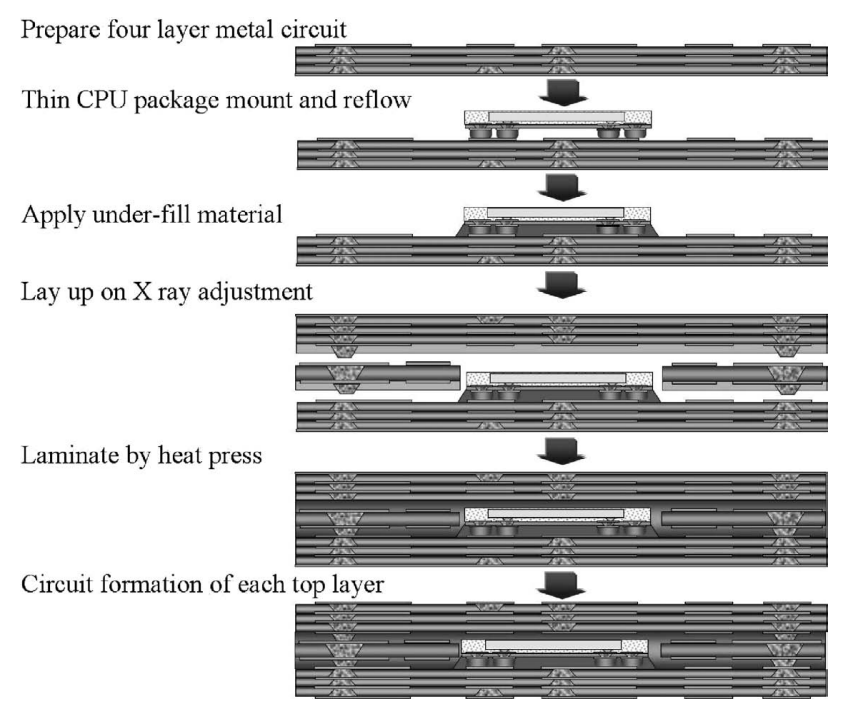

Fig. 3 CPU embed procedure with $\mathrm{B}^{2}$ it fabrication process.

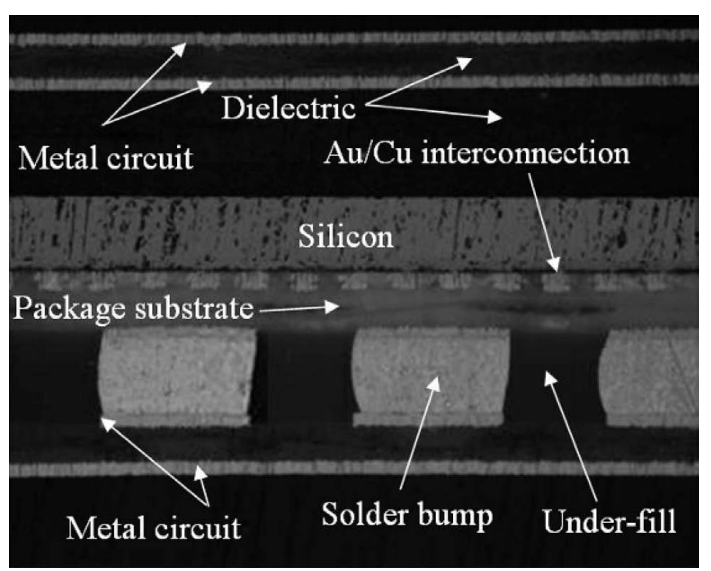

Fig. 4 Cross-section of thin silicon TEG-embedded PCB. mounted on the four-layered metal circuit PWB with leadfree solder-paste, and connection with this four layered metal circuit PWB is completed using IR-reflow. Thirdly, following this IR-reflow, electrical interconnection is completed by performing flux cleaning and under-fill resin injection. Fourthly, the two-layered core circuit PWB with device hole and four-layered metal circuit PWB are adjusted to this CPU-mounted four-layered metal circuit PWB using an X-ray camera alignment technology. After this alignment, the CPU embed process is completed thorough a laminating process, with the resin filling to the cavity part of the internal two-layered circuit PWB and the electrical connection between both PWB units done at the same time. Finally, the PWB fabrication is finished with a photolithography process to form metal circuits on both surfaces of this laminated PWB. In this way, the CPU embed procedure with $\mathrm{B}^{2}$ it fabrication technology has the feature of a small number of steps in the laminate process. Also, because pre-inspected circuit PWB units can be used for the CPU embed process, we can easily ensure highyield in the CPU-embedded PCB production.

Figure 4 shows a cross-sectional photograph of a completed PCB with an embedded thin silicon TEG chip of 400 pins. The $0.23 \mathrm{~mm}$ thin package and base circuit PWB are joined by lead-free solder bumps with a height of $0.1 \mathrm{~mm}$.

\subsection{Mechanical reliability of thin CPU-package- embedded PCB}

We examined three kinds of mechanical reliability tests for the thin CPU-package-embedded PCB with an eightlayered metal circuit configuration. Figure 5 shows the results of the thermal cycling, high-temperature operation life, and drop impact tests of this thin silicon embedded PCB.

All the mechanical reliability tests were conducted using

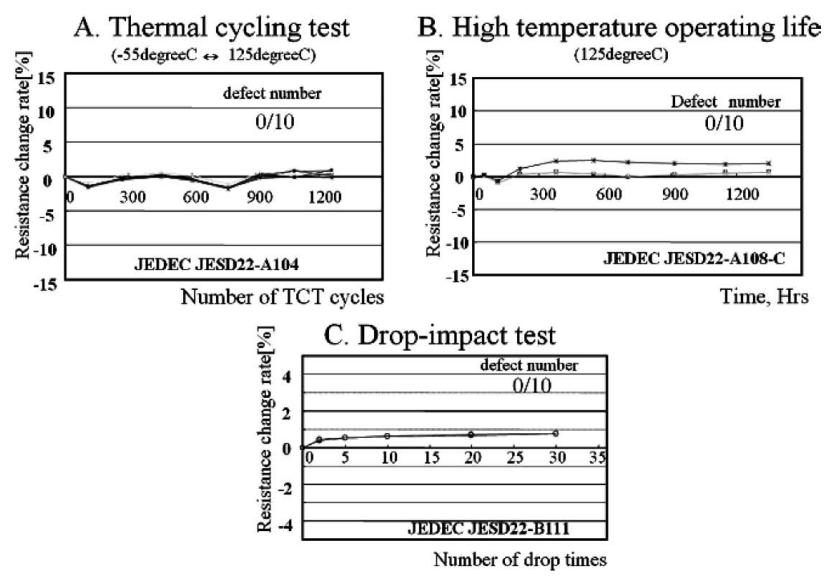

Fig. 5 Result of mechanical reliability test. 
ten finished PCBs with embedded silicon TEG. All mechanical reliability tests passed, satisfying the criteria of a rate of change within $10 \%$ after each test. These results indicate that thin CPU-package-embedded PCB with $\mathrm{B}^{2}$ it fabrication technology has sufficient reliability to operate in the field.

\section{Verification of memory access performance of CPU-embedded PCB substrate}

In this section, we introduce the verified results for the signal transmission performance of the SiP, which is composed of four surface mounted memories and one embedded CPU in a PWB. We mainly evaluated the signal integrities of data signal, crosstalk and switching noise using SPICE simulation technology. All of these validation case studies for electrical performances were executed using the reference model of a conventional side-by-side SiP structure.

\subsection{Three-dimensional floor plan for CPU-embedded SiP}

According to the design guidelines for DDR memory, the length of the address lines and data lines are required to be equal to the strobe signal in order to optimize the memory access performance. In particular, a layout design optimization that defines the characteristic impedance of the signal lines is very important to suppress the signal skew and crosstalk noise.

Figure 6 shows a schematic representation of the CPUembedded SiP model to verify the signal integrities, such as signal reflection, crosstalk noise, and simultaneous switching noise. In this defined analysis model, the I/O terminals of the embedded CPU and the surface-mounted memories are arranged to face each other in order to min-

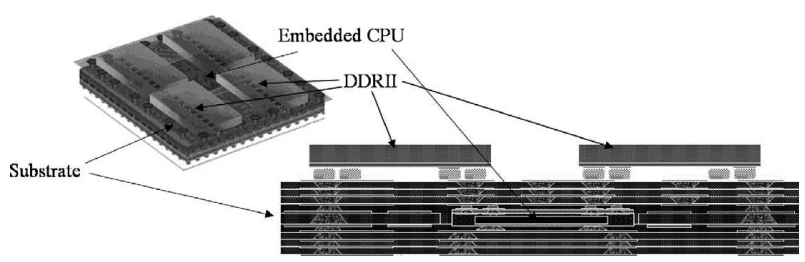

Fig. 6 Schematic view of CPU embedded SiP. imize the wiring-pattern lengths between them.

Figure 7 shows the practical consequences of the schematic layout design on studying electrical performances of CPU-embedded SiP and conventional side-by-side type SiP. The overall size of the conventional SiP, composed of a BGA-packaged CPU and memories, is $53 \times 53 \mathrm{~mm}$, and the $\mathrm{SiP}$ with embedded CPU is $24 \times 28 \mathrm{~mm}$. In this design consideration, the same critical design rule of PWB (i.e., L/S $=0.05 / 0.05 \mathrm{~mm}$ minimum) is applied to both the CPUembedded SiP and side-by-side SiP in order to equalize the process yield of PWB fabrication. From this layout design task, the CPU-embedded SiP shows an area reduction of about $70 \%$ against the side-by-side SiP.

\subsection{Signal and power integrity evaluations on CPU-} embedded SiP

In this section, we describe the analytical results of electrical performance on the CPU-embedded SiP using SPICE simulation technique in comparison to the side-by-side SiP. All signal integrity verification experiments were performed on a SPICE simulator, with accurate parasitic parameters of each wiring-pattern, which are extracted by the three-dimensional electro-magnetic field solver. Table1 shows the extracted parasitic parameters (i.e., R, L, G, and $C$ values) of typical signal wiring-pattern on each SiP. These parameters indicate combination values that contain the parasitic parameters of the CPU package and DDR package. It is particularly worth noting that all the values of the parasitic parameters on the CPU-embedded SiP are very small compared with the conventional side-by-side SiP except for capacitance.

Figure 8 shows the signal waveforms when DDRII mem-

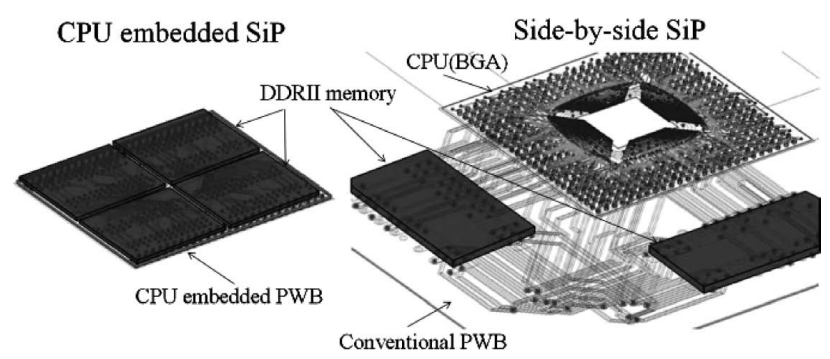

Fig. 7 Schematic of layout design.

Table 1 Parasitic parameters of each SiP.

\begin{tabular}{l|c|c|c|c|c|c}
\hline item & $\begin{array}{c}\text { Wiring length } \\
{[\mathrm{mm}]}\end{array}$ & $\begin{array}{c}\text { Propagation delay } \\
{[\mathrm{ps}]}\end{array}$ & $\begin{array}{c}\text { Resistance } \\
{[\mathrm{ohm}]}\end{array}$ & $\begin{array}{c}\text { Inductance } \\
{[\mathrm{nH}]}\end{array}$ & $\begin{array}{c}\text { Capacitance } \\
{[\mathrm{pF}]}\end{array}$ & $\begin{array}{c}\text { Conductance } \\
{[\mathrm{uS}]}\end{array}$ \\
\hline CPU embedded SiP & 9.5 & 96.2 & 0.4 & 2.2 & 4.5 & 282.0 \\
\hline Side-by-side SiP & 30.5 & 293.0 & 1.9 & 13.8 & 6.1 & 375.3 \\
\hline
\end{tabular}




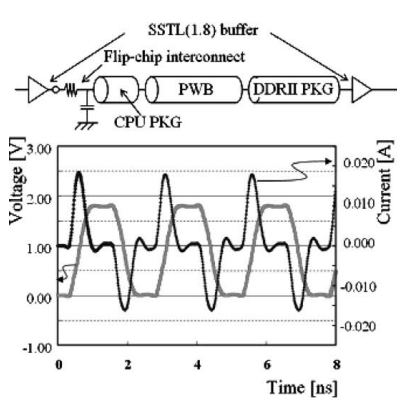

CPU embedded SiP

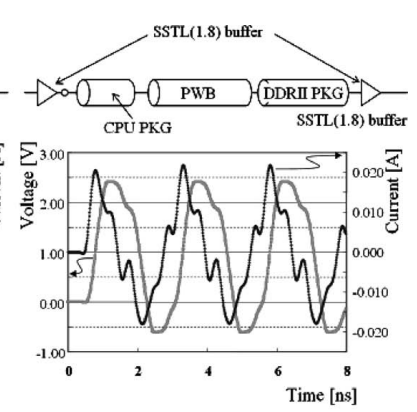

Side-by-side SiP
Fig. 8 Observed signal waveforms on DDR input edge.
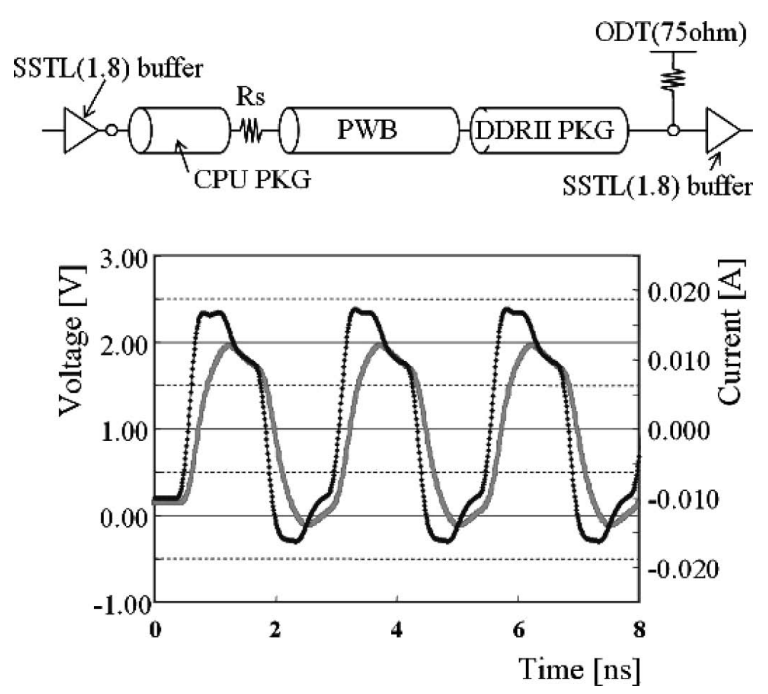

Fig. 9 Observed signal waveforms on DDR input edge of adjusted side-by-side SiP.

ories are driven from CPU I/O drivers. This simulation result is obtained without all of the passive components for adjusting the signal waveform. From this figure, we can easily recognize that large ringing and over/undershoot in the current and voltage waveforms occur in the side-byside SiP. These phenomena originate in the existence of large parasitic inductances in the wire and the impedance mismatch between the PWB wiring-pattern and the BGA package wiring-pattern. This result shows that it is difficult to drive DDRII memories in a side-by-side SiP without passive components for signal integrity adjustment. On the other hand, clear current and voltage waveforms can be obtained in the CPU-embedded SiP without passive components. Thus, excellent signal quality can be obtained in the CPU-embedded SiP, because it is possible to match the characteristic impedance of the signal wiring-pattern and the output impedance of the driver.

Figure 9 shows the result of the SPICE waveform on the optimized side-by-side SiP, when the on-die terminate function of DDRII is turned on and damping resistors are

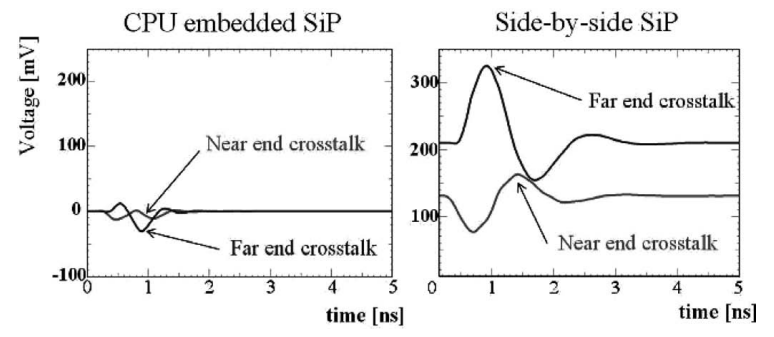

Fig. 10 Observed crosstalk noise voltage waveforms.

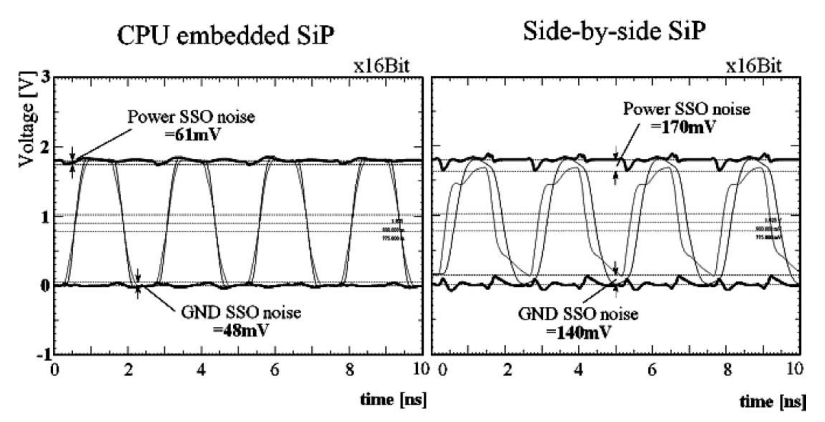

Fig. 11 SSO noise in common Vdd and Vss lines.

inserted on the wiring-pattern in series connection. From this result, the location of the damping resistors and the on-die terminate function are essential to reduce the overshoot/undershoot due to the excessive current and impedance mismatch in a side-by-side SiP.

Cross-talk noise will become a serious problem in highspeed memory bus lines composed of high-density parallel wiring-pattern. We therefore verified the resistance properties for crosstalk noise in the CPU-embedded SiP.

Figure 10 shows the observed result of cross-talk voltage on a quiet center position wiring pattern when seven peripheral wiring patterns are activated. The noise voltages in each $\mathrm{SiP}$ are not particularly problematic, but the values in the CPU-embedded SiP show extremely low levels in both near-end and far-end positions. Short wiring-pattern of the strip-structure with low characteristic impedance on the CPU-embedded SiP contributes to cross-talk noise suppression.

Figure 11 shows the observed voltage waveform of the common Vdd and Vss lines when sixteen I/Os are switching simultaneously. It is well known that SSO (Simultaneous Switching Output) noise voltage is proportional to the effective inductance of the common power-supply system and the driving power of the I/O buffer. We can see from this result that the voltage fluctuation of the power-supply system in the CPU-embedded SiP is very small compared with the side-by-side SiP. These excellent resistance properties for SSO noise depend on the small amount of dis- 
charged current and small effective inductance of common power supply via interconnection arranged in parallel. From this investigation, we checked that there are sufficient capabilities against simultaneous switching of sixteen-bit operation on this CPU-embedded SiP without embedded decouple-capacitors.

From these validation case studies with SPICE simulation, we verified the CPU-embedded SiP has better electrical performance than the side-by-side SiP. In addition, we checked that the CPU-embedded SiP has the advantage of reducing the number of passive components for adjusting the signal quality. As described above, the CPU-embedded $\mathrm{SiP}$ is the most effective solution to miniaturize SiP functions with a high-speed interface.

\section{Evaluation of signal communication perfor- mance in $\mathrm{I} / \mathrm{O}$ device embedded SiP}

In this section, we verify the signal-quality degradation of the output signals from the embedded CPU using an instrumental method. Communication signals from the embedded CPU to the external pads of the PCB should pass through many internal joint portions of the $\mathrm{PCB}$, because the embedded CPU and DDR memories were arranged closely opposite to each other in order to obtain the good electrical performance. Network communication performance with point to point on the distributed control system is a key factor in realizing high performance in humanoid robots. A high-speed LVDS (Low-voltage differential signal) communication interface is generally adopted to obtain good performance in the distributed control system of multiple processing modules. We therefore evaluate the communication performance with our CPUembedded SiP product.

\subsection{Outline of CPU I/O device-embedded SiP}

Figure 12 shows a cross-section of two types of CPU I/O device-embedded $\mathrm{SiP}$ in order to evaluate communication performance. One is a combination build-up PWB, consisting of a conventional PTH (Plated through hole) core and $\mathrm{B}^{2}$ it buildup layers. The conventional PTH core is laminated on both surfaces with two metal-layer circuits of $\mathrm{B}^{2}$ it

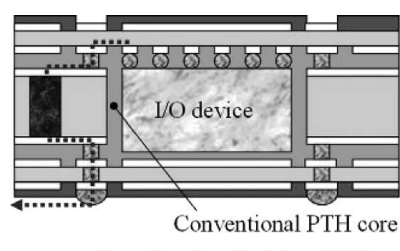

Combination buildup PWB

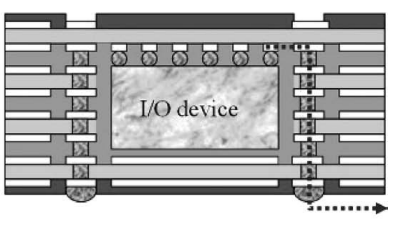

All-B ${ }^{2}$ it PWB
Fig. 12 Cross section of CPU I/O device embedded SiP. buildup, and electrical connections of both PCB units are made using Ag paste bumps. The second is a core-less buildup $\mathrm{PCB}$ with all-B ${ }^{2}$ it buildup layers. Differential signals from the embedded CPU I/O device to the external pads of the PCB are transmitted through fan-out wiring-pattern on Layer 2 and all internal vias as shown in Figure 12.

The power supply networks of this embedded CPU I/O device are wired with a stacked structure on the global wiring layers on the silicon die. Stacked structures can easily decrease the characteristic impedance of the power-supply wiring-pattern compared to coplanar structures. The low characteristic impedance of power-supply wiring-pattern enhances the signal integrity at the instant of high-speed switching.[4, 5] Therefore, we apply this principle (i.e., stacked structure of power supply wiring-pattern) to this device-embedded $\mathrm{SiP}$, in order to maintain the continuous power integrity. The embedded coplanar structure is applied for differential signal wiring-pattern to emphasize the differential coupling between $\mathrm{P}-\mathrm{N}$ channels. Furthermore, the shield plane of the internal layer is separated from the signal wiring-patterns to avoid leakage of electromagnetic coupling with this shield plane, because the discarded electromagnetic coupling with shield plane will lead to signal loss in the high-frequency operation. Figure 13 shows the stacked power supply wiring-patterns and embedded coplanar wiring-patterns for the differential signals. All the I/O wiring-patterns are tuned to $100 \mathrm{ohms}$ differential impedance with $0.09 \mathrm{~mm}$ width and $0.07 \mathrm{~mm}$ clearance. Moreover, the power and ground wiringpatterns are tuned to have less than $10 \mathrm{ohms}$ characteristic impedance with 0.5 to $1 \mathrm{~mm}$ wiring-pattern widths.

In the combination buildup PWB, the wiring-pattern length between the staggered lands on the PTH core is controlled to be short in order to prevent discarded signal reflection originating in the dogleg structure with this staggered land. Figure 14 shows a photograph of the CPU I/O device-embedded SiP used for this electrical evaluation. The upper left of Figure 14 shows a stereo-photograph of the CPU I/O device-embedded SiP, solder jointed with the

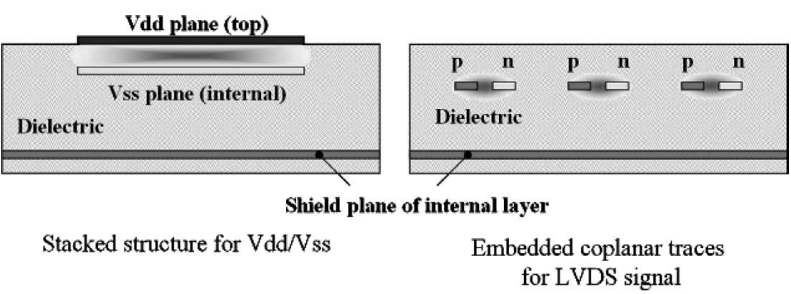

Fig. 13 Cross sections of I/O and power supply wiringpatterns. 


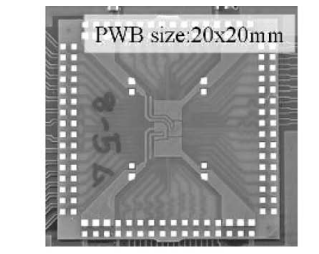

Stereo photograph of I/O device embedded PWB
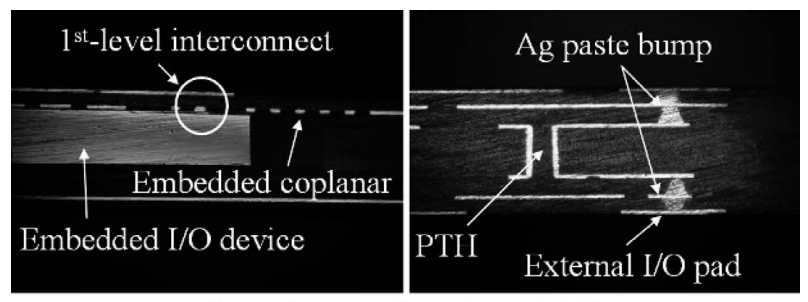

Combination build-up PWB
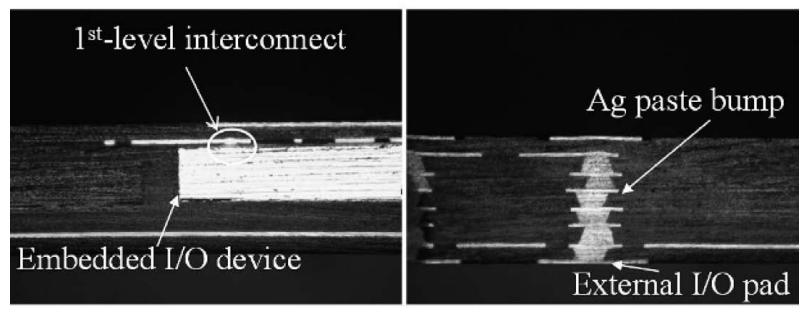

All-B ${ }^{2}$ it PWB

Fig. 15 Cross section of CPU I/O device embedded PWB.

evaluation PCB. This device-embedded SiP is $20 \mathrm{~mm} \times 20$ $\mathrm{mm}$ and the CPU I/O device is embedded in the center of this $\mathrm{PCB}$. The right picture shows an X-ray photograph of the CPU I/O device-embedded PCB. The block metal portions at the center are the common power-supply wiringpatterns, which are divided into four segments with stacked $\mathrm{Cu}$ metal formed on Layer 1 and Layer 2. The fine wiring-pattern portions with fan-out structures are the LVDS I/O signal wiring-patterns, and these differential characteristic impedances are adjusted to $100 \mathrm{ohms}$ with the structure of the embedded coplanar. The $0.3 \mathrm{~mm}$-thick embedded-CPU I/O device is located in the space bounded by the white line.

Figure 15 shows a cross-sectional view of two types of CPU I/O device embedded SiP. The major difference between them is the interconnection structure of the internal connection.

In the combination buildup PCB, a 0.25 mm-thick CPU I/ $\mathrm{O}$ device is embedded in the $0.25 \mathrm{~mm}$-thick PTH core. On the other hand, in the all- ${ }^{2}{ }^{2}$ PCB, the CPU I/O device is embedded in the 0.25 mm-thick four-layer $\mathrm{B}^{2}$ it buildup PWB. The finished combination buildup PCB and all- $\mathrm{B}^{2}$ it $\mathrm{PCB}$ are both $0.7 \mathrm{~mm}$ thick.

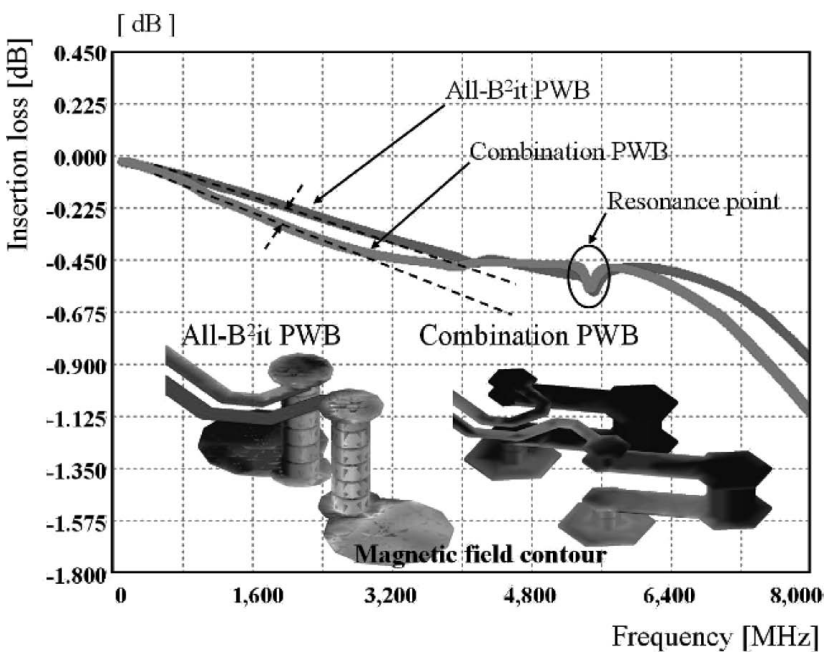

Fig. 16 Result of mix-mode insertion loss and static magnetic field of signal wiring-pattern.

\subsection{Electromagnetic field analysis of signal wiring}

Before checking the signal integrity using the instrumental method, we analyzed the wiring characteristics of each PWB of the SiP structure. Figure 16 shows a typical simulation result of mix-mode insertion loss of LVDS wiring in each PWB of the SiP. On both PWB models, the resonance points of insertion property are observed at about $5 \mathrm{GHz}$. These resonance points of insertion property are caused by the geometrical shape of the signal wiring; these phenomena occur regardless of the characteristics of each wiring pattern.

We can see that the all- $\mathrm{B}^{2}$ it PWB has better electrical mix-mode insertion-property performance in the 1 to 4 $\mathrm{GHz}$ frequency range. To figure out this difference in the insertion loss characteristic, we examined the magnetic field distribution analysis of the interconnect portion of each PWB model. The lower part of Figure 16 shows the magnetic field contour of the interconnect portion of each PWB model. In the all- $\mathrm{B}^{2}$ it PWB model, we can see that the mutual coupling strength of the magnetic field on the stacked via portion is strong everywhere. On the other hand, the magnetic field on the staggered land and PTH in the combination PWB model is very weak, and it varies widely and discontinuously. From this result, the cause of the large mix-mode insertion loss of differential coupling on the combination PWB model is attributed to the existence of the staggered interconnect structure with separately placed PTH in the core PWB. From these considerations, the device-embedded $\mathrm{PCB}$ with all- $\mathrm{B}^{2}$ it structure has superior transmission characteristics in the gigahertz frequency region. 


\subsection{Outline of measurement method of signal com- munication performance}

In this section, we explain the outline of the instrumental method to evaluate the integrity of the communication signal. We measured the communication signal waveforms with input reference signals of high-speed pulses and highspeed pseudo-random bit sequences (PRBS).

The CPU I/O device-embedded PCBs are mounted with solder balls on the four metal layered evaluation PCB, in order to observe the output signal from the embedded CPU I/O device. The I/O signal wiring patterns on the four metal layers of the PCB are designed according to the guidelines of micro-strip structures, controlled to $100 \mathrm{ohms}$ differential impedance. All the distances between the solder ball pads of the CPU I/O device-embedded PCB and the active probe terminals are adjusted to $20 \mathrm{~mm}$. Figure 17 shows a stereoscopic photograph of the CPU I/O device-embedded PCB with the evaluation PCB.

\subsection{Configuration of measurement system}

Figure 18 shows a schematic diagram for this experimental study of differential signal-transmission performance.

We installed a pulse-pattern generator (ANRITSUMP1761B) to generate an original high-speed signal and

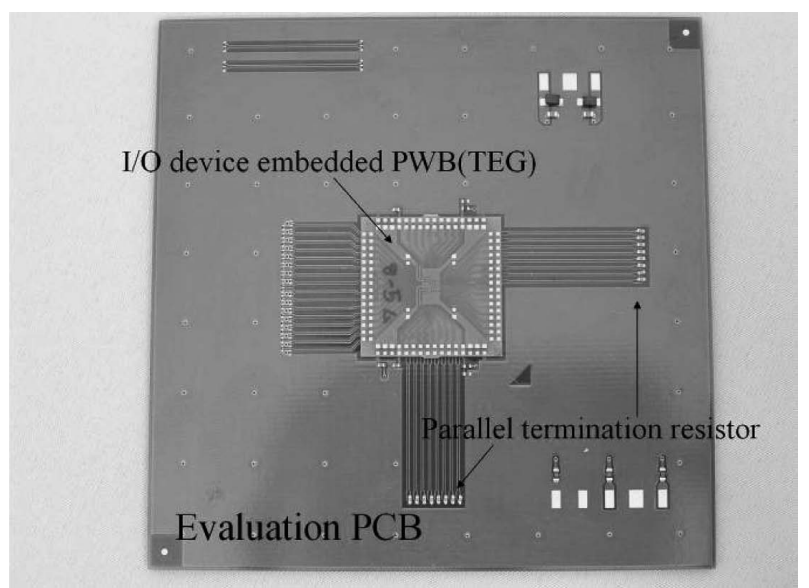

Fig. 17 Photograph of evaluation PCB with TEG.

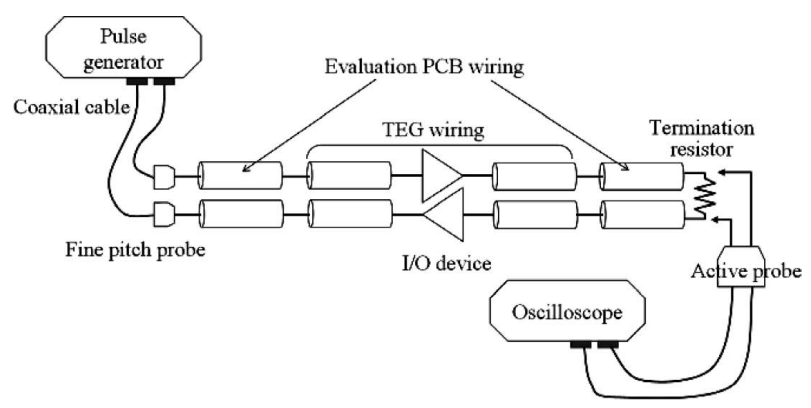

Fig. 18 Schematic diagram of measurement system.
PRBS. The output terminals from the pulse generator are connected to a fine pitch probe (Cascade Microtech-ACPGSG200) with coaxial cable. Wiring patterns to observe the output signals on the four metal layers of the PCB are terminated with parallel $100 \mathrm{ohms}$ resistors. The measurement nodes of the signal waveforms were arranged outside of these termination resistors and the waveform was observed with an oscilloscope (Agilent/86100A) through the active probe.

\subsection{Measurement study of signal transmission per-} formance

We evaluated the signal integrities of actual communicated signals with the LVDS driver of the embedded CPU I/O device, based on eye-diagram analysis. This instrumental method is the most effective to evaluate maximum communication capability, originated in the structural differences between the combination buildup PCB and all$\mathrm{B}^{2}$ it PCB. Figure 19 shows eye-diagrams of the combination buildup $\mathrm{PCB}$ with conventional core and the all- $\mathrm{B}^{2}$ it $\mathrm{PCB}$, respectively. The left side of this figure shows the data in the combination buildup $\mathrm{PCB}$ and the right side shows the all- $\mathrm{B}^{2}$ it $\mathrm{PCB}$. We can see that there are no differences of timing skew and voltage margin in each deviceembedded PCB up to the mid-range data rate (1 and 2 Gbps). On the other hand, at the high data rate of $3 \mathrm{Gbps}$, we can see that the voltage waveforms are distorted in unit interval on the combination buildup PCB. In addition, at the data rate of $3 \mathrm{Gbps}$ in this diagram, we find that the signal voltage in the combination buildup $\mathrm{PCB}$ is lower than in the all-B ${ }^{2}$ it PCB.

These results correspond to the behavior of the mixmode insertion loss analysis. It is considered that the sig-
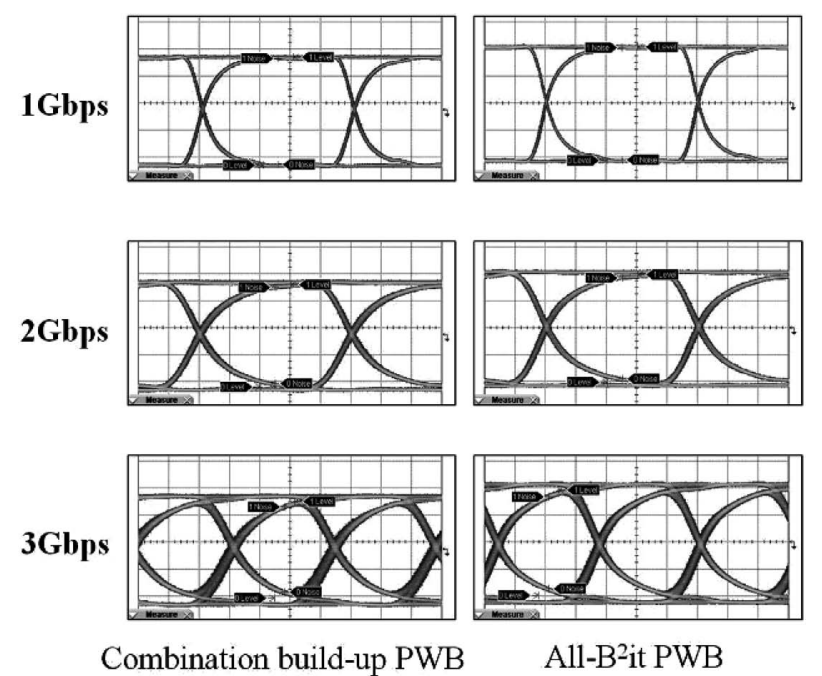

Fig. 19 Eye-diagram comparison of evaluation model. 


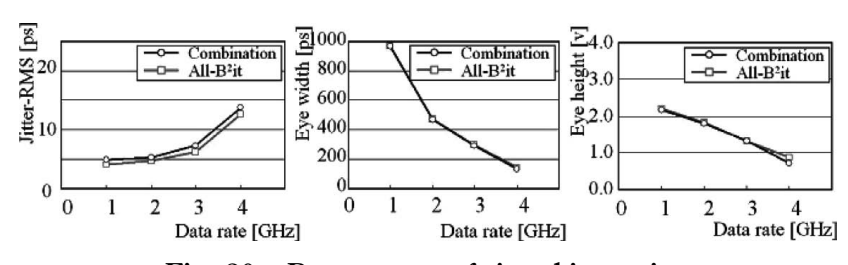

Fig. 20 Parameters of signal integrity.

nal loss the high data rate is caused by a discontinuous change of electro-magnetic coupling in the PTH portion with staggered land structure.

Figure 20 shows typical parameter values obtained from the eye-diagram measurement of this study. The JitterRMS values are extracted from the measured voltage waveform, defined as the shift length from 0 volt. Both the eye-width and eye-height become small at the high data rate in either CPU I/O device-embedded PCB. Moreover, there is no significant difference in the numerical values of these parameters. On the other hand, the values of eyejitter in the combination PCB are slightly larger than those in the all- $\mathrm{B}^{2}$ it $\mathrm{PCB}$ in all frequency regions. We note that both CPU I/O device-embedded PCB structures have sufficient communication signal-transmission performance corresponding to the data rate of $4 \mathrm{Gbps}$, because both peak to peak jitters are very small: 12.6 psec for the all- $\mathrm{B}^{2}$ it PCB and 13.8 psec for the combination PCB, respectively.

From these measurement studies and analyses, both structures of CPU I/O embedded PCB show sufficient communication signal transmission performance for the transmission speeds up to $4 \mathrm{Gbps}$.

\section{Conclusion}

We developed a high-density embedded device technol- ogy to miniaturize the motion-control CPU module for humanoid robots. From our investigation, it is concluded that a CPU-embedded PCB has excellent electrical performance, such as anti-signal reflection property, anti-cross-talk noise property, and resistance properties for simultaneous switching output noise. Furthermore, using an instrumental method, we verified that the CPU-embedded PCB has satisfactory signal-transmission performance, up to the data rate of $4 \mathrm{Gbps}$. However, we did not do an analysis of the power integrity for the CPU-core logic system in this article; we would like to investigate this remaining issue in the future.

\section{References}

[1] T. Motomura et al., "Development of Motion Control Module for Robots by SiP technology", International Conference on Electronics Packaging (ICEP2008) pp. 170-174.

[2] K. Sasaoka et al., "Development of PWB with Embedded Active Devices and Chip Passive Components", 17th Microelectronics Symposium (MES2007) pp. 159-162.

[3] S. Sagara et al., "Development of Thin LSI Embedded Circuit Boards", 41st International Symposium on Microelectronics (IMAPS2008) pp. 52-59.

[4] K. Hashimoto et al., "A study of Preferable Power Supply Method Focused on Consistent Power Wiring Configuration in GHz Digital System”, 3D-SIC2008 pp. 415-423.

[5] K. Hashimoto et al., "Development of Low Characteristic Impedance Transmission Line for Power Supply", 18th Microelectronics Symposium (MES2008). 